\title{
PRODUCTS OF INVOLUTION CLASSES IN INFINITE SYMMETRIC GROUPS
}

\author{
GADI MORAN
}

\begin{abstract}
Let $A$ be an infinite set. Denote by $S_{A}$ the group of all permutations of $A$, and let $R_{i}$ denote the class of involutions of $A$ moving $|A|$ elements and fixing $i$ elements $(0 \leq i \leq|A|)$. The products $R_{i} R_{j}$ were determined in [M1]. In this article we treat the products $R_{i_{1}} \cdots R_{i_{n}}$ for $n \geq 3$. Let INF denote the set of permutations in $S_{A}$ moving infinitely many elements. We show:

(1) $R_{i_{1}} \cdots R_{i_{n}}=S_{A}$ for $n \geq 4$.

(2)(a) $R_{i} R_{j} R_{k}=$ INF if $\{i, j, k\}$ contains two integers of different parity;

(b) $R_{i} R_{j} R_{k}=S_{A}$ if $i+j+k>0$ and all integers in $\{i, j, k\}$ have the same parity.

(3) $R_{0}^{3}=S_{A} \backslash E$, where $\theta \in E$ iff $\theta$ satisfies one of the following three conditions:

(i) $\theta$ moves precisely three elements.

(ii) $\theta$ moves precisely five elements.

(iii) $\theta$ moves precisely seven elements and has order 12 .

These results were announced in 1973 in [MO]. (1) and part of (2)(a) were generalized recently by Droste $[$ D1, D2].
\end{abstract}

0. Introduction. Let $S_{A}$ denote the symmetric group of all permutations of a set $A$. Elements of $A$ are referred to as "symbols". In 1972 Bertram [B] showed that if $A$ is countably infinite and $C$ is any conjugacy class (coc) in $S_{A}$ whose members move infinitely many symbols, then $C^{4}=S_{A}$. He conjectured that, moreover, for such a $\operatorname{coc} C, C^{3}=S_{A}$. In the same year, in the course of proving the nonbireflectionality of the automorphism groups of some infinite trees [M4], we were led to the study of products of involution classes in $S_{A}$ and found it possible to give a description of the product of any two such classes [M1], of any power of an involution class [M2, M3], and, for infinite $A$, of any product of involution classes [MO]. For $0 \leq i \leq|A|$, let $R_{i}=R_{i}(A)$ denote the set of permutations $\varphi$ of $A$ satisfying $\varphi^{2}=1$, fixing $i$ symbols and, if $A$ is infinite, moving $|A|$ symbols. A class of particular significance is $R_{0}$, the class of fixed-point-free-involutions, which turned out to be a counterexample to Bertram's conjecture ([MO, M1], and, later but independently, [DG]).

Our familiarity with products of cocs in the symmetric groups was significantly advanced during the 13 years since Bertram's conjecture and its counterexample (see [B, Bo, D1, D2, Dv, M5] where further reference is available). Recently, Droste [D2] combined the available data and proved the remarkable result that

Received by the editors April 17, 1986 and, in revised form, April 23, 1987.

1980 Mathematics Subject Classification (1985 Revision). Primary 20B07; Secondary 20B30, $20 \mathrm{E} 34$.

Supported in part by NSERC grant. 
$R_{0}=R_{0}(A)$ is in fact the only class in $S_{A}$ violating Bertram's conjecture, as he had earlier suspected [D1].

While proofs of some of the "Bar Mitsva" (13 years old) results announced in [MO] have been published [M1, M2] and some have been significantly generalized (see, e.g., [D1, D2]), others are still without published proof. Among these is the actual value of $R_{0}^{3}$. It is high time for this gap to be filled, and this is the main objective of the present paper. Let us start by reviewing the content of [MO].

Assume in the sequel that $A$ is an infinite set. In [MO] we announced three theorems, which we denote here by $\mathrm{T} 1, \mathrm{~T} 2$, and $\mathrm{T} 3$.

$\mathrm{T} 1$ deals with the products of two factors $R_{i} R_{j}$ in $S_{A}$. These products are determined in detail (for finite and infinite $A$ ) in [M1, Theorem 2.1]. T1 is then restated and proved as Corollary 2.5. (Theorem 2.1 in [M1] simplifies to Theorem 2.6 when $A$ is finite.)

T2 gives the precise value of products of three factors $R_{i} R_{j} R_{k}$ other than $R_{0}^{3}$, and is restated as (2) in the abstract. Among its immediate corollaries is $R_{i} R_{j} R_{k} R_{l}=$ $S_{A}$ for all $0 \leq i, j, k, l \leq|A|$, stated as (1) in the abstract. (Indeed, for any coc $D$ and any $\operatorname{coc} C \subseteq \mathrm{INF}$ we have $(D \cdot C) \cap \mathrm{INF} \neq \varnothing$; hence $D \subseteq C \cdot$ INF. Thus, (1) follows from (2) (and (3)).)

The part " $R_{i} R_{j} R_{k} \supseteq$ INF" of T2 was significantly generalized by Droste, who showed that in fact $C_{1} C_{2} C_{3} \supseteq$ INF holds for any three classes in $S_{A}$ moving $|A|$ elements [D1, Theorem 2]. It readily follows that $C_{1} C_{2} C_{3} C_{4}=S_{A}$ whenever $C_{i}$ are classes moving $|A|$ elements [D1, Corollary 5.1], which extends (1). See [D2] for other extensions of consequences of (2).

A complete proof of T2 is given in $\S 3$, where the part $R_{i} R_{j} R_{k} \supseteq$ INF is derived from a theorem of independent interest: Let $|A|=\aleph_{0}$. Then INF $=R_{k} C$ for $0 \leq k \leq \aleph_{0}$, where $C$ is the class of permutations having $\aleph_{0}$ infinite orbits and no finite orbit (Theorem 3.2). In fact, Droste showed [D1, Lemma 4.9] that INF $\subseteq D \cdot C$ for any $\operatorname{coc} D \subseteq \mathrm{INF}$ (Proposition 3.8). We mention in passing that $C$ was the first class shown to satisfy $C^{2}=S_{A}$ by A. B. Gray in his thesis in 1960 [G], and that $C$ is the only class of permutations with infinite orbits only that satisfies $C \subseteq R_{i} R_{j}$ for all $0 \leq i, j \leq \aleph_{0}[\mathbf{M 1}$, Corollary 2.3(1)]. See [D3] for a simple proof of Gray's result and other properties of $C$.

T3 determines the value of $R_{0}^{3}$, and is restated as (3) in the abstract. ${ }^{1}$ As a complete argument for it is a central goal of this paper, we first restate it in more detail. Consider the following four conditions on a permutation $\theta$ in $S_{A}$ :

$\left(3^{*}\right)$ : $\theta$ moves precisely three symbols.

$\left(5^{*}\right)$ : $\theta$ moves precisely five symbols, on which it acts as a 5 -cycle.

$\left(2^{*}+3^{*}\right)$ : $\theta$ moves precisely five symbols, on which it acts as a product of two disjoint cycles, one of length 2 and one of length 3 .

$\left(3^{*}+4^{*}\right)$ : $\theta$ moves precisely seven symbols, on which it acts as a product of two disjoint cycles, one of length 3 and one of length 4 .

Call $\theta \in S_{A}$ exceptional if it satisfies one of these conditions, and let $E=E(A)$ denote the set of all exceptional permutations $S_{A}$.

${ }^{1}$ In $\left[\right.$ MO, Theorem 3], read " $\xi$ of order 12 " instead of " $\xi^{12}=1 "$ ". 
T3-or (3) of our abstract-is restated as

THEOREM 0. Let $A$ be infinite, and let $\theta \in S_{A}$. The following are equivalent:

(1) $\theta \notin R_{0}^{3}$.

(2) $\theta \in E$; i.e., $\theta$ satisfies one of the conditions $\left(3^{*}\right),\left(5^{*}\right),\left(2^{*}+3^{*}\right),\left(3^{*}+4^{*}\right)$.

$\S \S 1$ and 2 are devoted to the proof of Theorem $0 .(2) \Rightarrow(1)$ is proved in $\S 1$, and $(1) \Rightarrow(2)$ is proved in $\S 2$. The condition that $A$ is infinite is essential in Theorem 0 , and $R_{0}^{3}$ for finite $A$ will be discussed elsewhere. We mention that while obviously $(2) \Rightarrow(1)$ holds for finite $A,(1) \Rightarrow(2)$ fails there, and there are many examples of finite $A$ 's and nonexceptional $\theta$ 's in $S_{A}$ which are not a product of three fixed-pointfree involutions. However, by Theorem 0 and the discussion preceding Proposition 1.2 in $\S 1$, for every such $A$ and $\theta$ there is a set $A^{\prime} \supseteq A$ of cardinality at most $2|A|$ such that the trivial extension $\theta^{\prime}$ of $\theta$ to $A^{\prime}\left(\theta^{\prime}\left(a^{\prime}\right)=a^{\prime}\right.$ for $\left.a^{\prime} \in A^{\prime} \backslash A\right)$ is in fact a product of three fixed-point-free involutions of $A^{\prime}$.

In $\S 4$ we discuss briefly products of involution classes in $S_{A}$ other than the $R_{i}$ 's and suggest (as problems) natural sequel to this work. We also provide four tables which complete the proof of $(2) \Rightarrow(1)$, in Theorem 0 , given in $\S 1$.

1. No exceptional permutation is in $R_{0}^{3}$. This section is devoted to the proof of the implication $(2) \Rightarrow(1)$ in Theorem 0 . We first develop some notation.

Let $\theta \in S_{A}, a \in A$. Then $\theta(a)$ denotes the value of $\theta$ at $a$ (so $(\theta \varphi)(a)=$ $\theta(\varphi(a))$; i.e., right acts first on a symbol), and $(a)_{\theta}$ is the $\theta$-orbit of $a$; that is $(a)_{\theta}=\left\{\theta^{m}(a): m \in \mathbb{Z}\right\}$, where $\mathbb{Z}=\{0,+1,-1,+2,-2, \ldots\}$ is the set of integers. For $1 \leq n \leq \aleph_{0}$ let $\bar{\theta}(n)$ denote the cardinality of the set of $\theta$-orbits of cardinality $n$. $\theta$ is called nicely even if $\bar{\theta}(n)$ is an even cardinal for all $1 \leq n \leq \aleph_{0}$ (where infinite cardinals are considered even). Let $\mathrm{NE}=\mathrm{NE}(A)$ denote the set of all nicely even permutations in $S_{A}$. The following two propositions hold for $A$ of arbitrary cardinality.

PROPOSITION $1.0[\mathrm{M1}] . R_{0}^{2}=\mathrm{NE}$.

Let $M(\theta)=\{a \in A: \theta(a) \neq a\}$ denote the support of $\theta$, and let $m(\theta)=|M(\theta)|$ denote its cardinality.

Proposition 1.1 [M1, Lemma A.3, p. 76]. Let $\varphi, \psi \in S_{A}, \theta=\varphi \psi$. The smallest subset $B$ of $A$ containing $M(\theta)$ which is both $\varphi$-and $\psi$-invariant is

$$
B=\bigcup_{a \in M(\theta)}(a)_{\varphi}=\bigcup_{a \in M(\theta)}(a)_{\psi}
$$

Assume that $\theta \in R_{0}^{3}$ and $M(\theta)$ contains $m$ symbols. By Proposition $1.0 \theta=\varphi \psi$, where $\varphi \in \mathrm{NE}(A), \psi \in R_{0}(A)$. Let $B$ be the smallest set containing $M(\theta)$ which is both $\varphi$ - and $\psi$-invariant. Since $\left|(a)_{\psi}\right|=2$ for all $a \in A$, we have $|B| \leq 2 m$, by Proposition 1.1. Let $\xi_{C}$ denote the restriction of $\xi \in S_{A}$ to a subset $C$ of $A$. Since $B$ is both $\varphi$ - and $\psi$-invariant, we have $\varphi_{B}, \psi_{B}, \theta_{B} \in S_{B}, \theta_{B}=\varphi_{B} \psi_{B}$; and with $B^{\prime}=A \backslash B, \varphi_{B^{\prime}}, \psi_{B^{\prime}}, \theta_{B^{\prime}} \in S_{B^{\prime}}, \theta_{B^{\prime}}=\varphi_{B^{\prime}} \psi_{B^{\prime}}$. But $\theta_{B^{\prime}}$ is the identity map of $B^{\prime}$, so $\varphi_{B^{\prime}}=\psi_{B^{\prime}}^{-1}, \bar{\varphi}_{B^{\prime}}(2)=\bar{\psi}_{B^{\prime}}(2), \bar{\varphi}_{B^{\prime}}(n)=\bar{\psi}_{B^{\prime}}(n)=0$ for $n \neq 2$ by $\psi \in R_{0}(A)$. Since $\varphi \in \mathrm{NE}$, we conclude that $\bar{\varphi}_{B}(n)=\bar{\varphi}_{B}(n)+\bar{\varphi}_{B^{\prime}}(n)=\bar{\varphi}(n)$ is an even cardinal for $n \neq 2$ (but $\bar{\varphi}_{B}(2)$ can be odd).

Assume now that $|A|=\aleph_{0}, \theta \in S_{A}$, and for some coinfinite $B, M(\theta) \subseteq B \subseteq A$, we have $\theta_{B}=\varphi_{B} \psi_{B}$, where $\psi_{B} \in R_{0}(B)$ and $\bar{\varphi}_{B}(n)$ is even for $n \neq 2$. Let 
$B^{\prime}=A \backslash B$ (so $\left|B^{\prime}\right|=\aleph_{0}$ ) and let $\varphi_{B^{\prime}}=\psi_{B^{\prime}} \in S_{B^{\prime}}$ be any fixed-point-free involution. Then if $\varphi, \psi \in S_{A}$ are defined by their restrictions $\varphi_{B}, \varphi_{B^{\prime}} ; \psi_{B}, \psi_{B^{\prime}}$ we have $\varphi \in \mathrm{NE}(A)$ (for " $\bar{\varphi}(2)$ is even" we need " $|A \backslash B|=\aleph_{0}$ ") and $\psi \in R_{0}(A)$, so $\theta=\varphi \psi$ implies $\theta \in R_{0}^{3}(A)$.

We have proved

Proposition 1.2. Let $A$ be infinite, $\theta \in S_{A}$, and let $m(\theta)=|M(\theta)|=m<\aleph_{0}$. Then the following are equivalent:

(i) $\theta \in R_{0}^{3}$ (where $R_{0}=R_{0}(A)$ ).

(ii) There is a set $B$ containing $M(\theta),|B| \leq 2 m$, and $\varphi, \psi \in S_{B}$ such that $\theta_{B} \psi=\varphi, \psi \in R_{0}(B)$, and $\bar{\varphi}(n)$ is an even integer for $n \neq 2$.

Let now $A$ be infinite, let $\theta \in S_{A}$ be an exceptional permutation, and let $m=$ $m(\theta)$. Then $m \leq 7$, and by Proposition $1.2 \theta \notin R_{0}^{3}$ follows once we can verify that for no set $B \supseteq A$ of cardinality at most $2 m \leq 14$ containing $M(\theta)$, we have $\theta_{B}=\varphi \psi$ for some $\varphi, \psi \in S_{B}, \psi \in R_{0}(B)$, and $\bar{\varphi}(n)$ even for $n \neq 2$. Thus, (2) $\Rightarrow(1)$ of Theorem 0 follows from

Proposition 1.3. Let $|B| \leq 14$, let $\theta \in S_{B}$ be exceptional, and let $\psi \in S_{B}$ be a fixed-point-free involution in $S_{B}$. Then $\varphi=\theta \psi$ satisfies: $\bar{\varphi}(n)$ is odd for some $n \neq 2$.

The proof of Proposition 1.3 involves the evaluation of finitely many products of an exceptional permutation by a fixed-point-free involution, and is readily done, e.g., by a graphical method introduced in [M2], explained in $\S 2$. The outcome of this computation, stated in the notation developed in $\S 2$, is given in $\S 4$ in Tables 1-4, whose content establishes Proposition 1.3.

2. Every nonexceptional permutation is in $R_{0}^{3}$. This section is devoted to the proof of the implication $(1) \Rightarrow(2)$ in Theorem 0 . We shall actually prove that $(1) \Rightarrow(2)$ when $\theta$ moves only finitely many symbols. If $\theta$ moves infinitely many symbols, i.e., $\theta \in I N F$, then $\theta \in R_{0}^{3}$ follows from (2)(a) in the abstract, which is proved in $\S 3$. Our goal then is to prove

THEOREM 2.0. Let $A$ be an infinite set, and let $\theta \in S_{A}$ move finitely many symbols. If $\theta \notin R_{0}^{3}$ then $\theta \in E$.

Our proof makes use of $[\mathbf{M 2}]$ and requires some more notation. Let $\mathbb{N}=$ $\{1,2, \ldots\}$ denote the set of positive integers, $\mathbb{N}^{+}=\mathbb{N} \cup\left\{\aleph_{0}\right\}$ and $\mathbb{N}_{0}=\mathbb{N} \cup\{0\}$. A type is a cardinal valued function defined on $\mathbb{N}^{+}$. We shall use small letters $r, s, t, \ldots$ to denote types, and boldface small letters $\mathbf{r}, \mathbf{s}, \mathbf{t}, \ldots$ to denote sets of types. The zero type $o$ is defined by $o(n)=0, n \in \mathbb{N}^{+}$, and for each $n \in \mathbb{N}^{+}, n^{*}$ is the type defined by $n^{*}(m)=\delta_{n m}, m \in \mathbb{N}^{+}$. The sum $\sum_{i \in I} t_{i}$ of a set of types $\left\{t_{i}: i \in I\right\}$ and the product $k \cdot t$ (or briefly, $k t$ ) of a type $t$ by a cardinal number $k$ are defined naturally by

$$
\left(\sum_{i \in I} t_{i}\right)(n)=\sum_{i \in I}\left(t_{i}(n)\right), \quad(k t)(n)=k(t(n)) \quad\left(n \in \mathbb{N}^{+}\right) .
$$

Thus, for any type $t$ we have

$$
t=\sum_{n \in N^{+}} t(n) \cdot n^{*}
$$


We note that the types with the addition as a binary operation and multiplication by a cardinal number form a semimodule over the semiring of cardinal numbers in a natural way.

For $\theta \in S_{A}$, the type $\bar{\theta}$ defined on $\mathbb{N}^{+}$in $\S 1$ by $\bar{\theta}(n)=$ cardinality of the set of $\theta$-orbits of cardinality $n$ is called the type of $\theta$, and $\theta$ is called a $t$-permutation if $\bar{\theta}=t$. Since $\theta, \theta^{\prime} \in S_{A}$ are conjugate if and only if $\bar{\theta}=\bar{\theta}^{\prime}$, types serve as convenient "names" for classes in the symmetric groups, and we proceed to use them to produce convenient notation for class invariants. For a type $t$ let

$$
\begin{aligned}
|t| & =\sum_{n \in N^{+}} n t(n) \quad \text { (cardinality of the domain), } \\
m(t) & =\sum_{1<n \in N^{+}} n t(n) \quad \text { (cardinality of the support). }
\end{aligned}
$$

$|t|$ is also called the cardinality of $t$, and $t$ is called finite if $|t|<\aleph_{0}$. $t$ is called finitary if $m(t)<\aleph_{0}$.

Define a three-place relation $P(r, s, t)$ on types as follows [M5]:

$P(r, s, t)$ iff there is a set $A$ and $\xi, \eta, \varsigma \in S_{A}$ such that $\bar{\xi}=r, \bar{\eta}=s, \bar{\zeta}=t$ and $\xi=\eta \varsigma$ (equivalently, $\left.\xi \eta \varsigma=1_{A}\right)$.

Thus, $P(r, s, t)$ implies $|r|=|s|=|t|$. The most useful properties of $P$ are [M5, Lemma 1]:

SYMMETRY: $P\left(t_{1}, t_{2}, t_{3}\right)$ if and only if $P\left(t_{i}, t_{j}, t_{k}\right)$ whenever $\{i, j, k\}=\{1,2,3\}$, SUPERADDITIVITY: $P\left(r_{i}, s_{i}, t_{i}\right)$ for all $i \in I$ implies

$$
P\left(\sum_{i \in I} r_{i}, \sum_{i \in I} s_{i}, \sum_{i \in I} t_{i}\right) .
$$

HoMOgeneity: $P(r, s, t)$ implies $P(k r, k s, k t)$ for every cardinal number $k$.

We now use $P$ to model the product of conjugacy classes in symmetric groups and, more generally, the products of conjugacy sets (subsets of $S_{A}$ closed under conjugacy in $S_{A}$ ) in the realm of types.

DEFinition 2.0. Let $\mathbf{s}, \mathbf{t}$ be sets of types. Define a set of types $\mathbf{s} \odot \mathbf{t}$ called the composition of $\mathbf{s}$ and $\mathbf{t}$ by

$$
r \in \mathbf{s} \odot \mathbf{t} \quad \text { iff } P(r, s, t) \text { holds for some } s \in \mathbf{s}, t \in \mathbf{t} .
$$

When no confusion may arise, it will be convenient to use the same symbol for a type and the singleton containing it. Thus, if $s, t$ are types we have

$$
s \odot t=\{s\} \odot\{t\} .
$$

The $n$th power $\mathbf{t}^{n}$ of a set of types $\mathbf{t}$ is defined inductively by $\mathbf{t}^{1}=\mathbf{t}, \mathbf{t}^{n+1}=$ $\mathbf{t}^{n} \odot \mathbf{t}$.

If $t$ is a type, we let $t^{n}=\{t\}^{n}$.

The class of sets of types forms a commutative semigroup with the composition operator $\odot$.

The addition of types extends naturally to sets of types by

$$
\mathbf{u}+\mathbf{v}=\{u+v: u \in \mathbf{u}, v \in \mathbf{v}\}
$$


Similarly, the product of a type by a cardinal number (scalar multiplication) extends naturally to sets of types by

$$
k \cdot \mathbf{u}=\{k u: u \in \mathbf{u}\} .
$$

The class of sets of types with addition + and scalar multiplication again forms a semimodule over the semiring of cardinal numbers, which carry also the semigroup operation of composition $\odot$. We shall use the following convention in forming expressions (terms) in this structure:

1. A type $t$ may always stand for the singleton $\{t\}$.

2. Priorities of operations in expressions involving $\cdot, \odot,+$ is in this order, unless indicated otherwise by bracketing.

Thus, for example,

$$
2 \cdot \aleph_{0}^{*} \odot \aleph_{0} \cdot\left\{3^{*}, 1^{*}+2^{*}\right\}+7^{*}=\left(\left(2 \cdot \aleph_{0}^{*}\right) \odot\left\{\aleph_{0} \cdot 3^{*}, \aleph_{0} \cdot 1^{*}+\aleph_{0} \cdot 2^{*}\right\}\right)+7^{*} \text {. }
$$

A most useful observation is

$$
u \odot v+u^{\prime} \odot v^{\prime} \subseteq\left(u+u^{\prime}\right) \odot\left(v+v^{\prime}\right),
$$

and, more generally,

$$
\sum_{i \in I} u_{i} \odot v_{i} \subseteq\left(\sum_{i \in I} u_{i}\right) \odot\left(\sum_{i \in I} v_{i}\right) .
$$

For $n \in \mathbb{N}^{+}$let $n^{\oplus}=\aleph_{0} \cdot n^{*}$. For a set of permutations $X$, let $\bar{X}=\{\bar{\xi}: \xi \in X\}$.

Thus, if $|A|=\aleph_{0}$, then $\bar{R}_{0}=\left\{2^{\oplus}\right\}=2^{\oplus}$ (by our convention that allows a type to stand for its singleton). We now define for $0 \leq i \leq \aleph_{0}$ a type $r_{i}$, so that $r_{i}=\bar{R}_{i}$, by

$$
r_{i}=i \cdot 1^{*}+\aleph_{0} \cdot 2^{*}=i \cdot 1^{*}+2^{\oplus}
$$

By Proposition 1.0 we have

$$
r_{0}^{2}=\left\{t:|t|=\aleph_{0}, t(n) \text { is even for all } n \in \mathbb{N}^{+}\right\}
$$

and

$$
r_{0}^{3}=r_{0}^{2} \odot r_{0}=\overline{R_{0}^{3}}=\left\{\bar{\theta}: \theta \in R_{0}^{3}\right\} .
$$

We note that by $r_{0} \in r_{0}^{2}, 1^{\oplus} \in r_{0}^{3}$ (i.e., the identity permutation $1_{A}$ of a countable set $A$ is a product of three fixed-point-free involutions), and so in Theorem 2.0 we may, with no loss of generality, assume that $A$ is countable. Thus, Theorem 2.0 is equivalent to

THEOREM 2.1. Let $|t|=\aleph_{0}, m(t)<\aleph_{0}, t \notin r_{0}^{3}$. Then $t \in 1^{\oplus}+\left\{3^{*}, 5^{*}, 2^{*}+\right.$ $\left.3^{*}, 3^{*}+4^{*}\right\}$.

Our next goal is to formulate a theorem on finite types, implying Theorem 2.1. First define some sets of finite types as follows.

$$
\begin{gathered}
\mathbf{r}_{0}=\left\{k \cdot 2^{*}: k \in \mathbb{N}_{0}\right\} \quad \text { (set of finite fixed-point-free involution types), } \\
\mathbf{n e}=r_{0}^{2} \quad \text { (set of finite NE types), } \\
\mathbf{r}_{00}=\mathbf{n e} \cup\left(\mathbf{n e}+2^{*}\right) \quad(\text { set of finite types } t \text { satisfying:t }(n) \text { is even for } n \neq 2), \\
\mathbf{r}_{000}=\mathbf{r}_{00} \odot \mathbf{r}_{0} .
\end{gathered}
$$


We have [M2, Lemma 1, p. 5]

(i) $\mathbf{r}_{0} \subseteq \mathbf{r}_{00} \subseteq \mathbf{r}_{000}$.

(ii) each of $\mathbf{r}_{0}, \mathbf{r}_{00}, \mathbf{r}_{000}$ is additively closed.

We now restate Proposition 1.2 as

Proposition 2.2. Let $t=1^{\oplus}+t_{0}$, where $\left|t_{0}\right|=m(t)=m<\aleph_{0}$. Then $t \in r_{0}^{3}$ if and only if $t_{0}+k \cdot 1^{*} \in \mathbf{r}_{000}$ for some $k \leq m$.

Thus, Theorem 2.1 (hence Theorem 2.0) follows from

THEOREM 2.3. Let $|t|<\aleph_{0}, t(1)=0$. The following are equivalent:

(a) For all $k \in \mathbb{N}_{0}, k \cdot 1^{*}+t \notin \mathbf{r}_{000}$,

(b) $t \in\left\{3^{*}, 5^{*}, 2^{*}+3^{*}, 3^{*}+4^{*}\right\}$.

PROOF OF THEOREM 2.3. (b) $\Rightarrow$ (a). Indeed, otherwise we have some $t \in$ $\left\{3^{*}, 5^{*}, 2^{*}+3^{*}, 3^{*}+4^{*}\right\}$ and some $k \in \mathbb{N}_{0}$ satisfying $k \cdot 1^{*}+t \in \mathbf{r}_{000}$, and so by Proposition 2.2, $m \cdot 1^{*}+t \in \mathbf{r}_{000}$, for some $m \leq 7$, contradicting Proposition 1.3.

The rest of this section is devoted to the proof of (a) $\Rightarrow(b)$. The argument for (a) $\Rightarrow$ (b) splits into five steps, the first three of which are essentially reproduced from [M2]. We first give

OUTLINE OF PROOF OF (a) $\Rightarrow$ (b).

First. Define a large additively closed subset $\mathbf{p}$ of $\mathbf{r}_{000}$ and a set of finite types called residua.

Second. Recall that every finite type $t$ admits a representation $t=t_{0}+t_{1}$ where $t_{0} \in \mathbf{p}$ and $t_{1}$ is a residuum.

Third. Describe a graphical method of establishing $t \in \mathbf{r}_{000}$.

Assume now that $|t|<\aleph_{0}, t(1)=0$ and for all $k \in \mathbb{N}_{0} t+k \cdot 1^{*} \notin \mathbf{r}_{000}$.

Fourth. Let $t=t_{0}+t_{1}$, where $t_{0} \in \mathbf{p}$ and $t_{1}$ is a residuum. Then $t_{1}=3^{*}$ or $t_{1}=5^{*}$.

Fifth. If $t_{1}=3^{*}$ then $t_{0} \in\left\{o, 2^{*}, 4^{*}\right\}$. If $t_{1}=5^{*}$ then $t_{0}=o$.

Obviously, this establishes (a) $\Rightarrow(\mathrm{b})$.

Let us turn to the details.

Define three families of finite types $\mathbf{f}_{i}, i=1,2,3$, as follows:

$$
\begin{array}{lll}
t \in \mathbf{f}_{1} & \text { iff } & t=2 \cdot n^{*} \text { for some } n \in \mathbb{N} . \\
t \in \mathbf{f}_{2} & \text { iff } & t=(2 n)^{*} \text { for some } n \in \mathbb{N}_{0} . \\
t \in \mathbf{f}_{3} & \text { iff } & t=(1+2 k)^{*}+(7+2 l)^{*} \text { for some } k, l \in \mathbb{N}_{0} .
\end{array}
$$

Let $p$ denote the additive closure of $f=f_{1} \cup f_{2} \cup f_{3}$; that is,

$$
t \in \mathbf{p} \text { iff } t \text { is a finite sum of members of } \mathbf{f} \text {. }
$$

( $\mathbf{p}$ is the class of types of the "proper permutations" in the terminology of [M2]; see [M2, Definition 4.5, p. 15].)

Notice that $o \in \mathbf{p}$ and that $|t|$ is even for every $t \in \mathbf{p}$, as this holds for any $t \in \mathbf{f}$. This follows also from

\section{Proposition 2.4 [M2, Proposition 4.6]. $\mathbf{p} \subseteq \mathbf{r}_{000}$.}

We call a finite type $t$ a residuum iff $t=t_{0}+t_{1}, t_{0} \in \mathbf{p} \Rightarrow t_{0}=o$. This definition immediately gives the following. 
PROPOSITION 2.5. Let $t$ be any finite type. Then $t=t_{0}+t_{1}$, where $t_{0} \in \mathbf{p}$ and $t_{1}$ is a residuum.

Notice that this representation is not unique. Indeed, if $t=1^{*}+3^{*}+5^{*}+7^{*}$, then $t=t_{0}+t_{1}=t_{0}^{\prime}+t_{1}^{\prime}$ are two distinct such representations, with $t_{0}=5^{*}+7^{*}$, $t_{1}=1^{*}+3^{*}, t_{0}^{\prime}=3^{*}+7^{*}, t_{1}^{\prime}=1^{*}+5^{*}$.

By inspecting the family $\mathbf{f}$ one easily sees

PROPOSITION 2.6. Let $t$ be a residuum. Then $t$ satisfies one of the following three conditions:

(0) $t=o$.

(1) $t=(2 n+1)^{*}$ for some $n \in \mathbb{N}_{0}$.

(2) $t \in\left\{1^{*}+3^{*}, 1^{*}+5^{*}, 3^{*}+5^{*}, 1^{*}+3^{*}+5^{*}\right\}$.

(See [M2, Definition 4.7 and Lemma 4.8, p. 16].)

Our argument in the sequel requires the verification of claims " $t \in \mathbf{r}_{000}$ " for various types. We will use the graphical method introduced in the appendix of [M2] which we reproduce here for the reader's convenience.

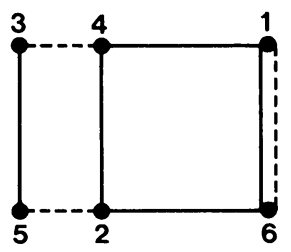

(a)
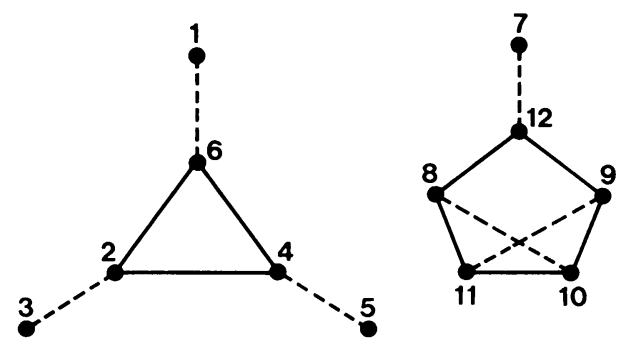

(b)

FIGURE 0

(a) Displays $\theta=\varphi \psi$, where

$$
\begin{aligned}
\theta & =(1)(2,3)(4,5,6)(7,8,9,10) \\
\varphi & =(3,5)(1,4,2,6)(7,10,9,8) \\
\psi & =(1,6)(2,5)(3,4)(7,9)(8,10) .
\end{aligned}
$$

Hence, $P\left(1^{*}+2^{*}+3^{*}+4^{*}, 2^{*}+2 \cdot 4^{*}, 5 \cdot 2^{*}\right)$, and so $1^{*}+2^{*}+3^{*}+4^{*} \in\left(2^{*}+2 \cdot 4^{*}\right) \odot 5 \cdot 2^{*} \subseteq$ $\mathbf{r}_{00} \odot \mathbf{r}_{0}=\mathbf{r}_{000}$.

(b) Displays $\varphi=\theta \psi$, where

$$
\begin{aligned}
\varphi & =(1,2,3,4,5,6)(7,8,9,10,11,12) \\
\theta & =(1)(3)(5)(7)(2,4,6)(8,11,10,9,12) \\
\psi & =(1,6)(2,3)(4,5)(7,12)(8,10)(9,11) .
\end{aligned}
$$

Hence, $P\left(2 \cdot 6^{*}, 4 \cdot 1^{*}+3^{*}+5^{*}, 6 \cdot 2^{*}\right)$, and so (by symmetry of $P$ ), $4 \cdot 1^{*}+3^{*}+5^{*} \in 2 \cdot 6^{*} \odot 6 \cdot 2^{*} \subseteq$ $\mathbf{r}_{00} \odot \mathbf{r}_{0}=\mathbf{r}_{000}$.

Let $t$ be a finite type. To show $t \in \mathbf{r}_{000}$, one has to produce permutations $\varphi, \psi$ of a set of cardinality $|t|$ with $\bar{\varphi} \in \mathbf{r}_{00}$ and $\bar{\psi} \in \mathbf{r}_{0}$ such that $\theta=\varphi \psi$ satisfies $\bar{\theta}=t$. We denote a $\varphi$-orbit of length $k$ greater than 2 as the set of vertices of a $k$-gon in the plane, whose sides, oriented positively (counterclockwise), describe 
the action of $\varphi$ on the vertices. $\varphi$-orbits of cardinality 2 are described as the endpoints of a line segment, and fixed points of $\varphi$ as isolated points. We describe the action of $\psi$ (who has only orbits of cardinality 2 ) by disjointed dashed segments, connecting pairs of points. The action of $\theta$ is obtained by following $\psi$-action first, then $\varphi$-action. To verify that indeed $t \in \mathbf{r}_{000}$, mark in order points as the $\theta$-action dictates. The disjoint-cycle decomposition obtained for $\theta$ should indicate that $\bar{\theta}=t$. (See Figure 0(a).) Equivalently, one can start with a description of the $\theta$-action by solid polygonal lines on the points, and the $\psi$-action by dashed line segment, and verify that $\varphi=\theta \psi$ satisfies $\bar{\varphi} \in \mathbf{r}_{00}$ (see Figure $0(\mathrm{~b})$ ).

We are now ready to proceed with the proof of $(\mathrm{a}) \Rightarrow(\mathrm{b})$.

Let $t$ be a fixed type satisfying $|t|<\aleph_{0}, t(1)=0$ and for all $k \in \mathbb{N}_{0} t+k \cdot 1^{*} \notin \mathbf{r}_{000}$. Let further $t=t_{0}+t_{1}$, where $t_{0} \in \mathbf{p}$ and $t_{1}$ is a residuum, as provided by Proposition 2.5 .

PROPOSITION 2.7. $t_{1}=3^{*}$ or $t_{1}=5^{*}$.

ProOF. We have to deny all other options for $t_{1}$ listed in Proposition 2.6.

$t_{1}=o$ is ruled out, as then $t \in \mathbf{p} \subseteq \mathbf{r}_{000}$.

$t_{1}=3^{*}+5^{*}$ is ruled out, as $4 \cdot 1^{*}+t_{1}=4 \cdot 1^{*}+3^{*}+5^{*} \in \mathbf{r}_{000}$ by Figure $0(\mathrm{~b})$, and so $4 \cdot 1^{*}+t=t_{0}+\left(4 \cdot 1^{*}+t_{1}\right) \in \mathbf{r}_{000}$ as $t_{0} \in \mathbf{p} \subseteq \mathbf{r}_{000}, 4 \cdot 1^{*}+t_{1} \in \mathbf{r}_{000}$, and $\mathbf{r}_{000}$ is additively closed. $t_{1} \in\left\{1^{*}+3^{*}, 1^{*}+5^{*}, 1^{*}+3^{*}+5^{*}\right\}$ is ruled out by $t(1)=t_{1}(1)=0$.

Thus, $t_{1}=(2 n+1)^{*}$ for some $n \in \mathbb{N}_{0}$. We need to show that $n=1$ or $n=2$. Indeed, $n=0$ is ruled out, as then $t_{1}=1^{*}$ and so $1^{*}+t=t_{0}+2 \cdot 1^{*} \in \mathbf{r}_{000}$. If $n \geq 3$, then $1^{*}+t_{1}=1^{*}+(7+2 k)^{*}$, where $k=n-3 \geq 0$, and so $1^{*}+t_{1} \in \mathbf{f}_{3} \subseteq \mathbf{p}$, and $1^{*}+t=t_{0}+\left(1^{*}+t_{1}\right) \in \mathbf{p}+\mathbf{p} \subseteq \mathbf{p} \subseteq \mathbf{r}_{000}$, i.e., $1^{*}+t \in \mathbf{r}_{000}$.

Thus $n=1$ or $n=2$ and $t_{1}=3^{*}$ or $t_{1}=5^{*}$.

Proposition 2.8. If $t_{1}=3^{*}$ then $t_{0} \in\left\{o, 2^{*}, 4^{*}\right\}$. If $t_{1}=5^{*}$ then $t_{0}=o$.

PROOF. We start with seven observations (1)-(7) of the form $s \in \mathbf{r}_{000}$, proved graphically by Figures $1-7$.

(1) $1^{*}+(3+2 k)^{*}+(6+2 l)^{*} \in \mathbf{r}_{000}$ for $k, l \in \mathbb{N}_{0}$. Indeed, by Figure 1 , $1^{*}+(3+2 k)^{*}+(6+2 l)^{*} \in\left(2 \cdot 1^{*}+(k+l) \cdot 2^{*}+2 \cdot 4^{*}\right) \odot(5+k+l) \cdot 2^{*} \subset \mathbf{r}_{000}$.

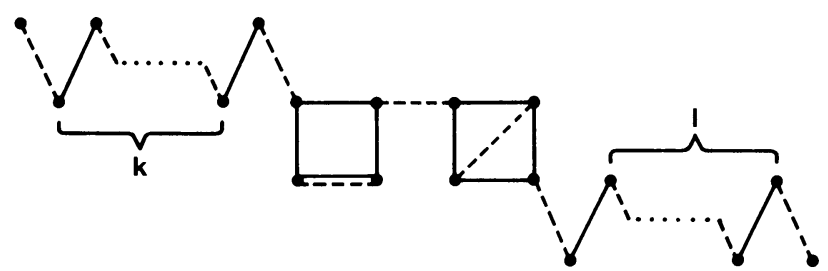

FIGURE 1

(2) $5 \cdot 1^{*}+3 \cdot 3^{*} \in \mathbf{r}_{000}$.

Indeed, by Figure 2,

$$
5 \cdot 1^{*}+3 \cdot 3^{*} \in\left(2^{*}+2 \cdot 6^{*}\right) \odot 7 \cdot 2^{*} \subseteq \mathbf{r}_{000} .
$$



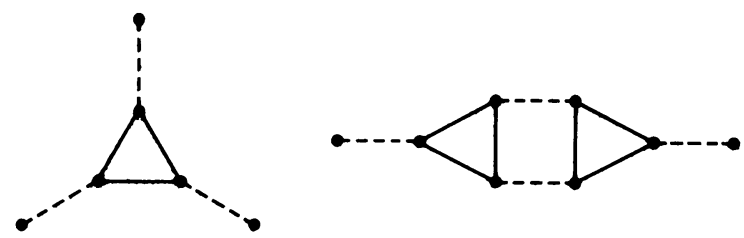

FIGURE 2

(3) $1^{*}+(3+2 k)^{*}+(3+2 l)^{*}+(5+2 m)^{*} \in \mathbf{r}_{000}$ for $k, l, m \in \mathbb{N}_{0}$.

Indeed, by Figure 3

$$
\begin{aligned}
& \mathbf{1}^{*}+(3+2 k)^{*}+(3+2 l)^{*}+(5+2 m)^{*} \\
& \quad \in\left(4 \cdot 1^{*}+(k+l+m) \cdot 2^{*}+2 \cdot 4^{*}\right) \odot(6+k+l+m) \cdot 2^{*} \subseteq \mathbf{r}_{000} .
\end{aligned}
$$
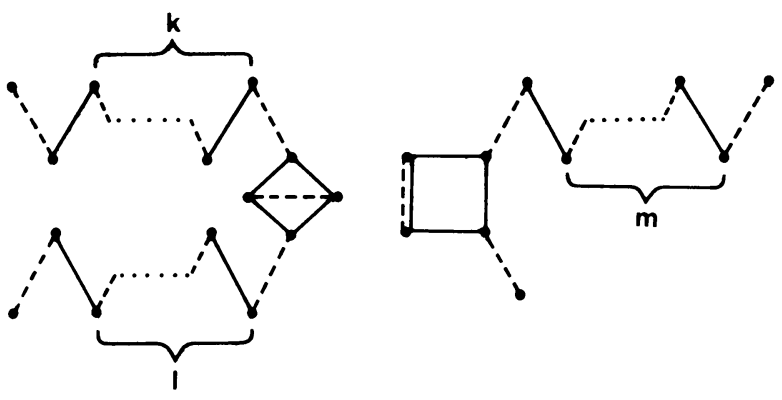

FIGURE 3

(4) $5 \cdot 1^{*}+2 \cdot 2^{*}+3^{*} \in \mathbf{r}_{000}$.

Indeed, by Figure 4 ,

$$
5 \cdot 1^{*}+2 \cdot 2^{*}+3^{*} \in 2 \cdot 6^{*} \odot 6 \cdot 2^{*} \subseteq \mathbf{r}_{000}
$$
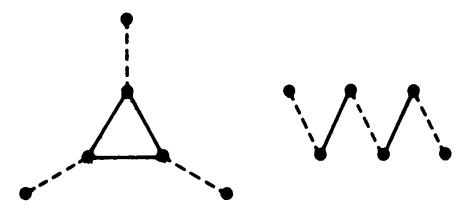

FiguRE 4

(5) $1^{*}+2^{*}+3^{*}+4^{*} \in \mathbf{r}_{000}$.

Indeed, see Figure 1(a).

(6) $7 \cdot 1^{*}+3^{*}+2 \cdot 4^{*} \in \mathbf{r}_{000}$.

Indeed, by Figure 5 ,

$$
7 \cdot 1^{*}+3^{*}+2 \cdot 4^{*} \in\left(2^{*}+2 \cdot 8^{*}\right) \odot 9 \cdot 2^{*} \subseteq \mathbf{r}_{000}
$$




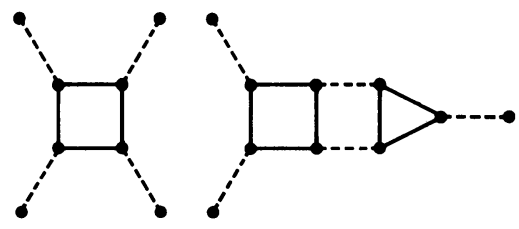

FIGURE 5

(7) $1^{*}+5^{*}+(2 n)^{*} \in \mathbf{r}_{000}$ for all $n \in \mathbb{N}$.

Indeed, for $n=1$ we have, by Figure 6 ,

$$
1^{*}+2^{*}+5^{*} \in\left(2^{*}+2 \cdot 3^{*}\right) \odot 4 \cdot 2^{*} \subseteq \mathbf{r}_{000}
$$

for $n=2$ we have, by Figure 7,

$$
1^{*}+4^{*}+5^{*} \in\left(2 \cdot 1^{*}+2 \cdot 4^{*}\right) \odot 5 \cdot 2^{*} \subseteq \mathbf{r}_{000}
$$

and for $n>2$, (7) follows from (1).

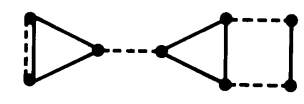

FIGURE 6

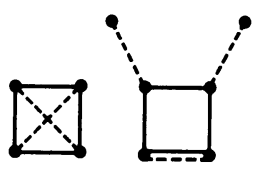

FIGURE 7

We proceed to prove Proposition 2.8. Thus, $t$ is a finite type satisfying $t(1)=0$, $t=t_{0}+t_{1}$, where $t_{0} \in \mathbf{p}$ and by Proposition $2.7 t_{1}=3^{*}$ or $t_{1}=5^{*}$, and in addition, for all $k \in \mathbb{N}_{0}, k \cdot 1^{*}+t \notin \mathbf{r}_{000}$.

Step 1. $t_{0}(2 n)=0$ for $n \geq 3$. (1)

Otherwise, let $t_{0}^{\prime}$ satisfying $t_{0}=t_{0}^{\prime}+(2 n)^{*}, n \geq 3$. Then $t_{0}^{\prime} \in \mathbf{p}$, and we have by

$$
1^{*}+t=1^{*}+(3+2 k)^{*}+(2 n)^{*}+t_{0}^{\prime} \in \mathbf{r}_{000}
$$

where $k=0$ if $t_{1}=3^{*}$, and $k=1$ if $t_{1}=5^{*}$.

Step 2. $t_{0}(2 n+1)=0$ for $n \geq 2$.

Otherwise, let $t_{0}=(5+2 m)^{*}+t_{0}^{\prime \prime}$, where $5+2 m=2 n+1, m \in \mathbb{N}_{0}$. Since $t_{0} \in \mathbf{p}, t_{0}$ is a finite sum of members of $\mathbf{f}$ and so $t_{0}=(5+2 m)^{*}+t_{0}^{\prime \prime}=(5+2 m)^{*}+\left(1+2 k^{\prime}\right)^{*}+t_{0}^{\prime}$, where $t_{0}^{\prime} \in \mathbf{p}$ and $\left(1+2 k^{\prime}\right)^{*}+(5+2 m)^{*} \in \mathbf{f}$. Since $t(1)=0$ we have $k^{\prime}>0$, and so $k^{\prime}=k+1$ for some $k \in \mathbb{N}_{0}$, and we have $t_{0}=(3+2 k)^{*}+(5+2 m)^{*}+t_{0}^{\prime}$ with $t_{0}^{\prime} \in \mathbf{p}$. Thus,

$$
t=t_{0}+t_{1}=(3+2 k)^{*}+(3+2 l)^{*}+(5+2 m)^{*}+t_{0}^{\prime},
$$

where $l=0$ if $t_{1}=3^{*}$ and $l=1$ if $t_{1}=5^{*}$. But then, by (3)

$$
1^{*}+t=1^{*}+(3+2 k)^{*}+(3+2 l)^{*}+(5+2 m)^{*}+t_{0}^{\prime} \in \mathbf{r}_{000} .
$$


Step 3. $t_{0}(n)=0$ for $n \neq 2,3,4$.

This follows from Steps 1,2 and $t(1)=0$.

Step 4. $t_{0}(2) \leq 1$ if $t_{1}=3^{*}, t_{0}(2)=0$ if $t_{1}=5^{*}$. Indeed:

1. If $t_{1}=3^{*}$ and $t_{0}(2) \geq 2$, let $t_{0}=2 \cdot 2^{*}+t_{0}^{\prime}$. Then $t_{0}^{\prime} \in \mathbf{p}$ and we have, by (4)

$$
5 \cdot 1^{*}+t=5 \cdot 1^{*}+2 \cdot 2^{*}+3^{*}+t_{0}^{\prime} \in \mathbf{r}_{000} \text {. }
$$

2. If $t_{1}=5^{*}$ and $t_{0}(2) \geq 1$, let $t_{0}=2^{*}+t_{0}^{\prime}$.

Then $t_{0}^{\prime} \in \mathbf{p}$ and we have by (7)

$$
1^{*}+t=1^{*}+2^{*}+5^{*}+t_{0}^{\prime} \in \mathbf{r}_{000}
$$

Step 5. $t_{0}(3)=0$.

Indeed, if $t_{0}(3)>0$ then $t_{0}(3) \geq 2$, as $t_{0} \in \mathbf{p}$ and by Step $3, t_{0}(2 n+1)=0$ for $n \neq 1$. Let $t_{0}=2 \cdot 3^{*}+t_{0}^{\prime}$. Then $t_{0}^{\prime} \in \mathbf{p}$, and we have:

If $t_{1}=3^{*}$, then by $(2)$

$$
5 \cdot 1^{*}+t=5 \cdot 1^{*}+3 \cdot 3^{*}+t_{0}^{\prime} \in \mathbf{r}_{000}
$$

If $t_{1}=5^{*}$, then by (3) (with $k=l=m=0$ )

$$
1^{*}+t=1^{*}+2 \cdot 3^{*}+5^{*}+t_{0}^{\prime} \in \mathbf{r}_{000}
$$

Step 6. $t_{0}(4) \leq 1$ if $t_{1}=3^{*}, t_{0}(4)=0$ if $t_{1}=5^{*}$.

Indeed, if $t_{1}=3^{*}$ and $t_{0}(4) \geq 2$, then $t_{0}=2 \cdot 4^{*}+t_{0}^{\prime}$, where $t_{0}^{\prime} \in \mathbf{p}$, and we have, by (6)

$$
7 \cdot 1^{*}+t=7 \cdot 1^{*}+3^{*}+2 \cdot 4^{*}+t_{0}^{\prime} \in \mathbf{r}_{000}
$$

while if $t_{1}=5^{*}$ and $t_{0}(4)>0$, then $t_{0}=4^{*}+t_{0}^{\prime}$, where $t_{0}^{\prime} \in \mathbf{p}$, and we have by (7)

$$
1^{*}+t=1^{*}+4^{*}+5^{*}+t_{0}^{\prime} \in \mathbf{r}_{000}
$$

Step 7. If $t_{1}=5^{*}$ then $t_{0}=o$.

This corollary of Steps 3-6 establishes Proposition 2.8 if $t_{1}=5^{*}$.

Step 8. If $t_{1}=3^{*}$ then $t_{0}(2)+t_{0}(4) \leq 1$.

By Steps 4 and $6 t_{0}(2), t_{0}(4) \leq 1$, so we have only to show that $t_{0}(2)=t_{0}(4)=1$ is impossible. Indeed, assume $t_{0}(2)=t_{0}(4)=1$. Then $t_{0}^{\prime} \in \mathbf{p}$, where $t_{0}^{\prime}$ is defined by $t_{0}=2^{*}+4^{*}+t_{0}^{\prime}$, and we have by (5)

$$
1^{*}+t=1^{*}+2^{*}+3^{*}+4^{*}+t_{0}^{\prime} \in \mathbf{r}_{000}
$$

Step 9. If $t_{1}=3^{*}$ then $t_{0} \in\left\{o, 2^{*}, 4^{*}\right\}$.

This corollary of Steps 3,5 and 8 establishes Proposition 2.8 if $t_{1}=3^{*}$.

The proof of Proposition 2.8 is complete, and Theorems 2.3, 2.1, 2.0 are proved.

3. The products $R_{i} R_{j} R_{k}, i+j+k>0$. This section is devoted to the proof of the statements $(2)(\mathrm{a})$ and $(2)(\mathrm{b})$ of the abstract. We first reduce the argument to two theorems that deal with countable $A$ (Theorems $3.2,3.3$ ).

Let $|A|=\aleph_{\nu}, \nu \geq 0$, and recall that

$$
\begin{aligned}
R_{i} & =R_{i}(A)=\left\{\varphi \in S_{A}: \varphi^{2}=1_{A}, \varphi \text { fixes } i \text { symbols and moves } \aleph_{\nu} \text { symbols }\right\}, \\
C & =C(A)=\left\{\theta \in S_{A}: \theta \text { has } \aleph_{\nu} \text { infinite orbits and no finite orbits }\right\} \\
\mathrm{INF} & =\operatorname{INF}(A)=\left\{\psi \in S_{A}: \psi \text { moves infinitely many symbols }\right\} .
\end{aligned}
$$

By [M1, Theorem 2.1] (or by simple direct argument, using Proposition 1.0 and [M1, Theorem 3.2(3)]) we have the following. 
Proposition 3.0. $C \subseteq R_{i} R_{j}$ for all $0 \leq i, j \leq \aleph_{\nu}$.

By [M1, Corollary 2.3(3); (3) on p. 77] we have

PROPOSITION 3.1. If $\{i, j, k\}$ has two integers of different parity, then $R_{i} R_{j} R_{k}$ $\subseteq \mathrm{INF}$.

We shall prove

THEOREM 3.2. Let $|A|=\aleph_{0}$. Then $\mathrm{INF}=C \cdot R_{k}$ for $0 \leq k \leq \aleph_{0}$.

THEOREM 3.3. Let $|A|=\aleph_{0}$. If $0 \leq i, j, k \leq \aleph_{0}, i+j+k>0$ and all integers in $\{i, j, k\}$ have the same parity, then $S_{A}=R_{i} R_{j} R_{k}$.

We now derive (2)(a) and (2)(b) using the terminology developed in $\S 2$.

For any ordinal $\nu$ let

$$
\operatorname{tp}_{\nu}=\left\{t:|t|=\aleph_{\nu}\right\}
$$

Thus $\mathbf{t p}_{\nu}=\bar{S}_{A}$ whenever $|A|=\aleph_{\nu}$.

$$
\inf _{\nu}=\left\{t:|t|=\aleph_{\nu}, m(t) \geq \aleph_{0}\right\} .
$$

Thus, $\inf _{\nu}=\overline{\operatorname{INF}(A)}$ whenever $|A|=\aleph_{\nu}$.

$$
r_{i, \nu}=i \cdot 1^{*}+\aleph_{\nu} \cdot 2^{*} .
$$

Thus $r_{i, 0}=r_{i}$, and $r_{i, \nu}=\bar{\psi}$ for any $\psi \in R_{i}(A)$ if $|A|=\aleph_{\nu}$.

We have

(i) $\mathbf{t p}_{\nu}=r_{k, \nu} \odot r_{k, \nu}$ for $\aleph_{0} \leq k \leq \aleph_{\nu}$ if $\nu>0$ [M2, Corollary 2.5].

(ii) inf $_{0} \subseteq r_{i, 0} \odot r_{j, 0} \odot r_{k, 0}$ for $0 \leq i, j, k \leq \aleph_{0}$, by Proposition 3.0 and Theorem 3.2 .

(iii) inf $_{0} \subseteq \aleph_{0}^{\oplus} \odot r_{k, 0}$ for $0 \leq k \leq \aleph_{0}$, by Theorem 3.2 .

(iv) $\aleph_{0}^{\oplus} \in r_{i, 0} \odot r_{j, 0}$ for $0 \leq i, j \leq \aleph_{0}$, by Proposition 3.0.

(v) $r_{k, \nu} \in r_{i, \nu} \odot r_{j, \nu}$ iff all integers in $\{i, j, k\}$ have the same parity.

(v) follows from [M1, Theorem 2.1], but we sketch a direct proof here:

1. If, say, $i+j<\aleph_{0}$ and $i+j \equiv 1(\bmod 2)$, then any $t \in r_{i, \nu} \odot r_{j, \nu}$ satisfies $t\left(\aleph_{0}\right)>0$ by [M1, Corollary 2.3(3)], so for all $k, r_{k, \nu} \notin r_{i, \nu} \odot r_{j, \nu}$.

2. If all integers in $\{i, j, k\}$ have the same parity, then $r_{k, \nu} \in r_{i, \nu} \odot r_{j, \nu}$. Indeed, w.l.o.g. $i \leq j, k$ and so, by assumption, $j=i+2 u, k=i+2 v$ for some $0 \leq u, v \leq \aleph_{\nu}$. Let $A=A_{0} \cup A_{1}$, where $\left|A_{0}\right|=i,\left|A_{1}\right|=\aleph_{\nu}$ and let $A_{1}=B \cup \dot{\cup} C \dot{0}$ where $|B|=2 u$, $|C|=2 v,|D|=\aleph_{\nu}$. Then one easily defines $\varphi, \psi \in S_{A}$ such that $A_{0}, B, C, D$ are $\varphi$ and $\psi$-invariant, $\varphi_{A_{0}}=\psi_{A_{0}}=1_{A_{0}}, \varphi_{A_{1}}$ is a fixed-point-free involution, $\psi_{B}=1_{B}$, $\psi_{C}=\varphi_{C}$, and $\psi_{D}$ is a fixed-point-free involution, as is $\varphi_{D} \psi_{D}$. Then $\bar{\varphi}=r_{i, \nu}$, $\bar{\psi}=r_{j, \nu}$ and $\overline{\varphi \psi}=r_{k, \nu}$.

With no loss of generality, assume $0 \leq i \leq j \leq k \leq \aleph_{\nu}$ in the sequel.

PROPOSITION 3.4. If $\nu>0, k \geq \aleph_{0}$, and $j \geq \aleph_{0}$ or $j<\aleph_{0}$ and $i \equiv j(\bmod 2)$, then $R_{i} R_{j} R_{k}=S_{A}$.

PROOF. We have to show $\mathbf{t p}_{\nu}=r_{i, \nu} \odot r_{j, \nu} \odot r_{k, \nu}$. But under these assumptions, we have $r_{k, \nu} \in r_{i, \nu} \cdot r_{j, \nu}$ by (v), and $r_{k, \nu} \cdot r_{k, \nu}=\mathbf{t p}_{\nu}$ by (i). Thus,

$$
\mathbf{t p}_{\nu}=r_{k, \nu} \cdot r_{k, \nu} \subseteq r_{i, \nu} \cdot r_{j, \nu} \cdot r_{k, \nu} \subseteq \mathbf{t p}_{\nu}
$$


Proposition 3.5. INF $\subseteq R_{i} R_{j} R_{k}$ for all $0 \leq i, j, k \leq|A|$.

Proof. For $\nu=0$ this holds by Theorem 3.2 and Proposition 3.0, so assume $\nu>0$. We have to show inf $\subseteq r_{i, \nu} \odot r_{j, \nu} \odot r_{k, \nu}$.

Case 1. $k \geq \aleph_{0}$. By Proposition 3.4, INF $\subseteq R_{i} R_{j} R_{k}$ if $j \geq \aleph_{0}$, so assume $j<\aleph_{0}$. Thus, we have $r_{i, \nu}=r_{i, 0}+r_{0, \nu}, r_{j, \nu}=r_{j, 0}+r_{0, \nu}$. Hence, by (iv)

$$
\begin{aligned}
\aleph_{0}^{\oplus}+r_{k, \nu} & \in r_{i, 0} \odot r_{j, 0}+r_{0, \nu} \odot r_{0, \nu} \subseteq\left(r_{i, 0}+r_{0, \nu}\right) \odot\left(r_{j, 0}+r_{0, \nu}\right) \\
& =r_{i, \nu} \odot r_{j, \nu} .
\end{aligned}
$$

Also, by (i), (iii) and $r_{k, \nu}=r_{0,0}+r_{k, \nu}$,

$$
\inf _{\nu}=\inf _{0}+\operatorname{tp}_{\nu} \subseteq \aleph_{0}^{\oplus} \odot r_{0,0}+r_{k, \nu} \odot r_{k, \nu} \subseteq\left(\aleph_{0}^{\oplus}+r_{k, \nu}\right) \odot r_{k, \nu} .
$$

Thus

$$
\inf _{\nu} \subseteq\left(\aleph_{0}^{\oplus}+r_{k, \nu}\right) \odot r_{k, \nu} \subseteq r_{i, \nu} \odot r_{j, \nu} \odot r_{k, \nu} .
$$

Case 2. $k<\aleph_{0}$. First note that by (ii), inf $\mathbf{p}_{\mathbf{0}} \subseteq r_{0,0}^{3}$. Since $1^{\oplus} \in r_{0,0}^{3}$ as well, and $r_{0, \nu}=\aleph_{\nu} \cdot r_{0,0}$, we conclude that $\inf _{\nu} \subseteq r_{0, \nu}^{3}$. Indeed, any $t \in \inf _{\nu}$ is representable as $t=\sum_{i \in I} t_{i}$, where $|I|=\aleph_{\nu}$ and $t_{i} \in \inf _{\mathbf{0}} \cup\left\{1^{\oplus}\right\}$; so $t_{i} \in r_{0,0}^{3}$ for all $i \in I$ and we have

$$
t=\sum_{i \in I} t_{i} \in \aleph_{\nu} \cdot\left(r_{0,0}^{3}\right) \subseteq\left(\aleph_{\nu} \cdot r_{0,0}\right)^{3}=r_{0, \nu}^{3} .
$$

By $r_{i, \nu}=r_{i, 0}+r_{0, \nu}, r_{j, \nu}=r_{j, 0}+r_{0, \nu}$ and (iv) we obtain

$$
\begin{aligned}
\aleph_{0}^{\oplus}+r_{0, \nu}^{2} & \subseteq r_{i, 0} \odot r_{j, 0}+r_{0, \nu} \odot r_{0, \nu} \subseteq\left(r_{i, 0}+r_{0, \nu}\right) \odot\left(r_{j, 0}+r_{0, \nu}\right) \\
& =r_{i, \nu} \odot r_{j, \nu} .
\end{aligned}
$$

Hence, by $r_{k, \nu}=r_{k, 0}+r_{0, \nu}$ and (ii)

$$
\begin{aligned}
\inf _{0}+\inf _{\nu} & \subseteq \aleph_{0}^{\oplus} \odot r_{k, 0}+r_{0, \nu}^{2} \odot r_{0, \nu} \\
& \subseteq\left(\aleph_{0}^{\oplus}+r_{0, \nu}^{2}\right) \odot\left(r_{k, 0}+r_{0, \nu}\right)=\left(\aleph_{0}^{\oplus}+r_{0, \nu}^{2}\right) \odot r_{k, \nu} .
\end{aligned}
$$

Similarly,

$$
\inf _{\mathbf{0}}+\aleph_{\nu} \cdot 1^{*} \subseteq\left(\aleph_{0}^{\oplus}+\nu_{0, \nu}^{2}\right) \odot \nu_{k, \nu}
$$

Also,

$$
\inf _{\nu}=\left(\inf _{0}+\inf _{\nu}\right) \cup\left(\inf _{0}+\aleph_{\nu} \cdot 1^{*}\right)
$$

so

$$
\inf _{\nu} \subseteq\left(\aleph_{0}^{\oplus}+r_{0, \nu}^{2}\right) \odot \nu_{k, \nu}
$$

Thus, again

$$
\inf _{\nu} \subseteq\left(\aleph_{0}^{\oplus}+r_{0, \nu}^{2}\right) \odot r_{k, \nu} \subseteq r_{i, \nu} \odot r_{j, \nu} \odot r_{k, \nu} .
$$

PROPOSITION 3.6. $R_{i} R_{j} R_{k}=\mathrm{INF}$ if $\{i, j, k\}$ contains two integers of different parity.

ProOF. By Proposition 3.5 $R_{i} R_{j} R_{k} \supseteq \mathrm{INF}$, and by Proposition $3.1 R_{i} R_{j} R_{k} \subseteq$ INF. 
Proposition 3.7. $R_{i} R_{j} R_{k}=S_{A}$ if $i+j+k>0$ and all integers in $\{i, j, k\}$ have the same parity.

Proof. For $\nu=0$ this identity holds by Theorem 3.3, so assume $\nu>0$. We have to show $\mathbf{t p}_{\nu} \subseteq r_{i, \nu} \odot r_{j, \nu} \odot r_{k, \nu}$. By Proposition 3.4 we may further assume $k<\aleph_{0}$. Hence

$$
r_{i, \nu}=r_{i, 0}+r_{0, \nu}, \quad r_{j, \nu}=r_{j, 0}+r_{0, \nu}, \quad r_{k, \nu}=r_{k, 0}+r_{0, \nu} .
$$

For any ordinal $\nu$, let

$$
\mathbf{e}_{\nu}=\aleph_{\nu} \cdot 1^{*}+\left\{3^{*}, 5^{*}, 2^{*}+3^{*}, 3^{*}+4^{*}\right\} .
$$

By Proposition 3.5 and Theorem $2.0 r_{0, \nu}^{3}=\operatorname{tp}_{\nu} \backslash \mathbf{e}_{\nu}$. Hence, by $\mathbf{e}_{\nu}=\mathbf{e}_{0}+\aleph_{\nu} \cdot 1^{*} \subseteq$ $\mathbf{t} \mathbf{p}_{0}+r_{0, \nu}^{3}$ and $r_{0, \nu}^{3} \subseteq \mathbf{t p}_{0}+r_{0, \nu}^{3}$, we have $\mathbf{t p}_{0}+r_{0, \nu}^{3}=\mathbf{t} \mathbf{p}_{\nu}$. Since by Theorem 3.3 $r_{i, 0} \odot r_{j, 0} \odot r_{k, 0}=\mathbf{t p}_{0}$, we have

$$
\begin{aligned}
\mathbf{t p}_{\nu} & =\mathbf{t p}_{0}+r_{0, \nu}^{3}=r_{i, 0} \odot r_{j, 0} \odot r_{k, 0}+r_{0, \nu} \odot r_{0, \nu} \odot r_{0, \nu} \\
& \subseteq\left(r_{i, 0}+r_{0, \nu}\right) \odot\left(r_{j, 0}+r_{0, \nu}\right) \odot\left(r_{k, 0}+r_{0, \nu}\right) \\
& =r_{i, \nu} \odot r_{j, \nu} \odot r_{k, \nu} . \quad \square
\end{aligned}
$$

(2)(a) and (2)(b) are Propositions 3.6 and 3.7, respectively.

We now prove Theorems 3.2 and 3.3. Recall that for $0 \leq i \leq \aleph_{0}, r_{i}=i \cdot 1^{*}+$ $\aleph_{0} \cdot 2^{*}=i \cdot 1^{*}+2^{\oplus}$.

ProOF OF TheOREM 3.2. Obviously $C \cdot R_{k} \subseteq$ INF for all $0 \leq k \leq \aleph_{0}$. Indeed, if $\varphi \in S_{A}$ is finitary, i.e., moves finitely many symbols, $\theta \in C$, then $\psi=\theta \varphi$ must have infinite orbits, and so $\psi \notin R_{k}$ for all $0 \leq k \leq \aleph_{0}$. Thus, $C \cdot R_{k}$ contains no finitary permutations; i.e., $C \cdot R_{k} \subseteq \mathrm{INF}$.

INF $\subseteq C \cdot R_{k}$ for all $0 \leq k \leq \aleph_{0}$ is a consequence of Droste's result:

Proposition 3.8 [D1, LEMMA 4.9]. INF $\subseteq C \cdot D$ for any class $D \subseteq$ INF.

ProOF OF TheOREM 3.3. By Proposition 3.5 it is enough to establish: If

Proposition 3.9. Let fin $=\left\{t: m(t)<|t|=\aleph_{0}\right\}$, and let $0 \leq i \leq j \leq k \leq \aleph_{0}$.

(*) $\{i, j, k\}$ has no two integers of different parity,

(**) $0<k$,

then fin $\subseteq r_{i} \odot r_{j} \odot r_{k}$.

Proposition 3.9 is established via a sequence of reductions.

3.9.1. $r_{l} \in r_{i} \odot r_{j}$ iff $\{i, j, l\}$ does not contain an even integer and an odd integer.

3.9 .1 is a restatement of $(\mathrm{v})$.

3.9.2. fin $\subseteq r_{\aleph_{0}} \odot r_{\aleph_{0}}$. Indeed,

$$
\text { fin } \subseteq\left\{t:|t|=\sum_{n \in N^{+}} t(n)=\aleph_{0}\right\}=r_{\aleph_{0}} \odot r_{\aleph_{0}},
$$

where the last equality is a restatement of [M1, Corollary 2.4].

3.9.3. We may assume $k<\aleph_{0}$. Indeed, by $(*)$ and 3.9.1, $r_{k} \in r_{i} \odot r_{j}$, so $r_{k}^{2}=r_{k} \odot r_{k} \subseteq r_{i} \odot r_{j} \odot r_{k}$. If $k=\aleph_{0}$ then, by 3.9.2, fin $\subseteq r_{i} \odot r_{j} \odot r_{k}$. 
3.9.4. $r_{0}^{3} \cap$ fin $\subseteq r_{i} \odot r_{j} \odot r_{k}$. Indeed, $r_{i}=r_{i}+r_{0}, r_{j}=r_{j}+r_{0}, r_{k}=r_{k}+r_{0}$; hence

$$
r_{i} \odot r_{j} \odot r_{k}=\left(r_{i}+r_{0}\right) \odot\left(r_{j}+r_{0}\right) \odot\left(r_{k}+r_{0}\right) \supseteq r_{i} \odot r_{j} \odot r_{k}+r_{0}^{3} .
$$

But by $(*)$ and 3.9.1 $r_{k} \in r_{i} \odot r_{j}$, so $1^{\oplus} \in r_{k} \odot r_{k} \subseteq r_{i} \odot r_{j} \odot r_{k}$. Hence $1^{\oplus}+r_{0}^{3} \subseteq$ $r_{i} \odot r_{j} \odot r_{k}$. But by fin $\cap r_{0}^{3} \subseteq 1^{\oplus}+r_{0}^{3}$ we have $r_{0}^{3} \cap$ fin $\subseteq r_{i} \odot r_{j} \odot r_{k}$.

3.9.5. Let $n^{+}=n^{*}+1^{\oplus}\left(n \in \mathbb{N}^{+}\right)$. Let $\mathbf{e}=\mathbf{e}_{0}$ denote the set of four types $\left\{3^{+}, 5^{+}, 2^{+}+3^{+}, 3^{+}+4^{+}\right\}$. If $\mathbf{e} \subseteq r_{i} \odot r_{j} \odot r_{k}$ then fin $\subseteq r_{i} \odot r_{j} \odot r_{k}$.

Indeed, by Theorem 2.0, fin $\backslash \mathbf{e} \subseteq r_{0}^{3}$ so by 3.9.4, fin $\backslash \mathbf{e} \subseteq r_{i} \odot r_{j} \odot r_{k}$.

3.9.6. For any $k \in \mathbb{N}$ we have $3^{+}, 5^{+} \in r_{k} \odot r_{k}, 2^{+}+3^{+}, 3^{+}+4^{+} \in r_{k+2} \odot r_{k}$. Indeed, $3^{*} \in\left(1^{*}+2^{*}\right)^{2}, 5^{*} \in\left(1^{*}+2 \cdot 2^{*}\right)^{2}$ by

$$
\begin{aligned}
(1,2,3) & =[(1,2)(3)][(1),(2,3)], \\
(1,2,3,4,5) & =[(1,2)(3,5)(4)][(1),(2,5)(3,4)] .
\end{aligned}
$$

Also, $1^{\oplus} \in t^{2}$ whenever $|t|=\aleph_{0}$, so, since $k-1 \geq 0$,

$$
\begin{aligned}
3^{+}= & 3^{*}+1^{\oplus} \in\left(1^{*}+2^{*}\right) \odot\left(1^{*}+2^{*}\right)+r_{k-1} \odot r_{k-1} \subseteq\left(1^{*}+2^{*}+r_{k-1}\right)^{2}=r_{k}^{2}, \\
5^{+}= & 5^{*}+1^{\oplus} \in\left(1^{*}+2 \cdot 2^{*}\right) \odot\left(1^{*}+2 \cdot 2^{*}\right) \\
& +r_{k-1} \odot r_{k-1} \subset\left(1^{*}+2 \cdot 2^{*}+r_{k-1}\right)^{2}=r_{k}^{2} .
\end{aligned}
$$

Similarly,

$$
\begin{aligned}
2^{*}+3^{*} & \in\left(3 \cdot 1^{*}+2^{*}\right) \odot\left(1^{*}+2 \cdot 2^{*}\right) \\
\left(3^{*}+4^{*}\right) & \in\left(3 \cdot 1^{*}+2 \cdot 2^{*}\right) \odot\left(1^{*}+3 \cdot 2^{*}\right)
\end{aligned}
$$

by

$$
\begin{aligned}
& (1,2,3)(4,5)=[(1,2)(3)(4)(5)][(1)(2,3)(4,5)] \\
& (1,2,3)(4,5,6,7)=[(1,2)(3)(4,6)(5)(7)][(1)(2,3)(4,5)(6,7)]
\end{aligned}
$$

Thus

$$
\begin{aligned}
2^{+}+3^{+} & =\left(2^{*}+3^{*}\right)+1^{\oplus} \in\left(3 \cdot 1^{*}+2^{*}\right) \odot\left(1^{*}+2 \cdot 2^{*}\right)+r_{k-1} \odot r_{k-1} \\
& \subseteq\left(3 \cdot 1^{*}+2^{*}+r_{k-1}\right) \odot\left(1^{*}+2 \cdot 2^{*}+r_{k-1}\right)=r_{k+2} \odot r_{k}
\end{aligned}
$$

and

$3^{+}+4^{+}=3^{*}+4^{*}+1^{\oplus} \in\left(3 \cdot 1^{*}+2 \cdot 2^{*}+r_{k-1}\right) \odot\left(1^{*}+2 \cdot 2^{*}+r_{k-1}\right)=r_{k+2} \odot r$.

3.9.7. $\mathbf{e} \subseteq r_{i} \odot r_{j} \odot r_{k}$ whenever $k \in \mathbb{N}$. Indeed, by 3.9.6 e $\subseteq r_{k}^{2} \cup\left(r_{k+2} \odot r_{k}\right)$, and by $(*)$ and 3.9.1 $r_{k}, r_{k+2} \in r_{i} \odot r_{j}$, so $\mathbf{e} \subseteq r_{i} \odot r_{j} \odot r_{k}$.

PROOF OF PROPOSITION 3.9. If $k=\aleph_{0}$, fin $\subseteq r_{i} \odot r_{j} \odot r_{k}$ by 3.9.2. If $0<k<\aleph_{0}$, fin $\subseteq r_{i} \odot r_{j} \odot r_{k}$ by 3.9.5 and 3.9.7.

The proof of Theorem 3.3 is complete.

4. Odds and ends. 1. We mention some problems suggested by the results of this paper.

We did not evaluate products of involution classes in $S_{A}$ involving classes moving less than $|A|$ elements. Some such products are, however, readily available. For instance, if $|A|=\aleph_{\nu}$ and $R$ is an involution class moving $\aleph_{\tau}$ symbols where $0 \leq$ $\tau<\nu$, then $R^{2}$ is the group $S_{A}^{\tau+1}$ of permutations of $A$ moving at most $\aleph_{\tau}$ symbols (this follows from [M1, Corollary 2.5] and mentioned in [MO]); it follows that if 
$R^{\prime}$ is another class moving $\aleph_{\sigma}$ symbols, $\tau \leq \sigma \leq \nu$, then $R R^{\prime}=S_{A}^{\tau+1} \cdot R^{\prime}$. Thus, products of arbitrary number of involution classes moving infinitely many symbols, can be evaluated easily (compare [M1, Theorem A.1, p. 75]). Thus, the following seems to be interesting (and, we believe, tractable):

Problem 1. Let $|A|=\aleph_{0}$. Determine the products $I_{n_{1}} \cdots I_{n_{k}}$, where $I_{n}$ is an involution class in $S_{A}$ whose members move $2 n$ symbols.

For $k=2$ this problem is solved in [M1]. The powers $I_{n}^{k}$ can be recovered from [M2, M3].

Theorem 3.2 and Proposition 3.8 suggest

Problem 2. Determine the set $K$ all cocs $E \subseteq$ INF satisfying $E \subseteq D_{1} \cdot D_{2}$ for all $\operatorname{cocs} D_{1}, D_{2} \subseteq$ INF.

$C \in K$ by Droste's result [D1, Lemma 4.9 (Proposition 3.8)].

2. We complete the proof of the implication of $(2) \Rightarrow(1)$ in $\S 1$ by displaying the products of the exceptional classes in $S_{A}$ by the class of fixed-point-free involutions of $A$, where $m \leq|A| \leq 2 m,|A|$ even, and $m$ is the number of symbols moved by a member of the exceptional class under discussion. The proof is completed in verifying that each permutation in each such product has an odd number of orbits of length $n$ for some $n \neq 2$.

This can be checked in the following listing of the twelve relevant sets of types (see §2). The set is denoted on top of a column, under which its members are listed.

TABLE 1

\begin{tabular}{ll}
$\left(1^{*}+3^{*}\right) \odot 2 \cdot 2^{*}$ & $\left(3 \cdot 1^{*}+3^{*}\right) \odot 3 \cdot 2^{*}$ \\
\hline $1^{*}+3^{*}$ & $1^{*}+2^{*}+3^{*}$ \\
& $6^{*}$ \\
\hline
\end{tabular}

TABLE 2

\begin{tabular}{lll}
$\left(1^{*}+5^{*}\right) \odot 3 \cdot 2^{*}$ & $\left(3 \cdot 1^{*}+5^{*}\right) \odot 4 \cdot 2^{*}$ & $\left(5 \cdot 1^{*}+5^{*}\right) \odot 5 \cdot 2^{*}$ \\
\hline $1^{*}+2^{*}+3^{*}$ & $1^{*}+2 \cdot 2^{*}+3^{*}$ & $1^{*}+3 \cdot 2^{*}+3^{*}$ \\
$2 \cdot 1^{*}+4^{*}$ & $2 \cdot 1^{*}+2^{*}+4^{*}$ & $2 \cdot 1^{*}+2 \cdot 2^{*}+4^{*}$ \\
$6^{*}$ & $2^{*}+6^{*}$ & $2 \cdot 2^{*}+6^{*}$ \\
& $1^{*}+7^{*}$ & $1^{*}+2^{*}+7^{*}$ \\
& $3^{*}+5^{*}$ & $2^{*}+3^{*}+5^{*}$ \\
& & $10^{*}$ \\
\hline
\end{tabular}

TABLE 3

\begin{tabular}{lll}
$\left(1^{*}+2^{*}+3^{*}\right) \odot 3 \cdot 2^{*}$ & $\left(3 \cdot 1^{*}+2^{*}+3^{*}\right) \odot 4 \cdot 2^{*}$ & $\left(5 \cdot 1^{*}+2^{*}+3^{*}\right) \odot 5 \cdot 2^{*}$ \\
\hline $3 \cdot 1^{*}+3^{*}$ & $3 \cdot 1^{*}+2^{*}+3^{*}$ & $3 \cdot 1^{*}+2 \cdot 2^{*}+3^{*}$ \\
$1^{*}+5^{*}$ & $1^{*}+2^{*}+5^{*}$ & $1^{*}+2 \cdot 2^{*}+5^{*}$ \\
$2^{*}+4^{*}$ & $2 \cdot 2^{*}+4^{*}$ & $3 \cdot 2^{*}+4^{*}$ \\
& $1^{*}+3^{*}+4^{*}$ & $1^{*}+2^{*}+3^{*}+4^{*}$ \\
& $8^{*}$ & $2^{*}+8^{*}$ \\
& & $4^{*}+6^{*}$ \\
\hline
\end{tabular}


TABLE 4

\begin{tabular}{llll}
$\left(1^{*}+3^{*}+4^{*}\right) \odot 4 \cdot 2^{*}$ & $\left(3 \cdot 1^{*}+3^{*}+4^{*}\right) \odot 5 \cdot 2^{*}$ & $\left(5 \cdot 1^{*}+3^{*}+4^{*}\right) \odot 6 \cdot 2^{*}$ & $\left(7 \cdot 1^{*}+3^{*}+4^{*}\right) \odot 7 \cdot 2^{*}$ \\
\hline $3 \cdot 1^{*}+2^{*}+3^{*}$ & $3 \cdot 1^{*}+2 \cdot 2^{*}+3^{*}$ & $3 \cdot 1^{*}+3 \cdot 2^{*}+3^{*}$ & $3 \cdot 1^{*}+4 \cdot 2^{*}+3^{*}$ \\
$1^{*}+3^{*}+4^{*}$ & $1^{*}+2^{*}+3^{*}+4^{*}$ & $1^{*}+2 \cdot 2^{*}+3^{*}+4^{*}$ & $1^{*}+3 \cdot 2^{*}+3^{*}+4^{*}$ \\
$1^{*}+2^{*}+5^{*}$ & $1^{*}+2 \cdot 2^{*}+5^{*}$ & $1^{*}+3 \cdot 2^{*}+5^{*}$ & $1^{*}+4 \cdot 2^{*}+5^{*}$ \\
$2 \cdot 2^{*}+4^{*}$ & $3 \cdot 2^{*}+4^{*}$ & $4 \cdot 2^{*}+4^{*}$ & $5 \cdot 2^{*}+4^{*}$ \\
$2 \cdot 1^{*}+6^{*}$ & $2 \cdot 1^{*}+2^{*}+6^{*}$ & $2 \cdot 1^{*}+2 \cdot 2^{*}+6^{*}$ & $2 \cdot 1^{*}+3 \cdot 2^{*}+6^{*}$ \\
$8^{*}$ & $2^{*}+8^{*}$ & $2 \cdot 2^{*}+8^{*}$ & $3 \cdot 2^{*}+8$ \\
$1^{*}+3 \cdot 3^{*}$ & $1^{*}+2^{*}+3 \cdot 3^{*}$ & $1^{*}+2 \cdot 2^{*}+3 \cdot 3^{*}$ \\
& $2 \cdot 1^{*}+3^{*}+5^{*}$ & $2 \cdot 1^{*}+2^{*}+3^{*}+5^{*}$ & $2 \cdot 1^{*}+2 \cdot 2^{*}+3^{*}+5^{*}$ \\
$2^{*} 1^{*}+2^{*}+6^{*}$ & $2 \cdot 1^{*}+2 \cdot 2^{*}+6^{*}$ & $2 \cdot 1^{*}+3 \cdot 2^{*}+6^{*}$ \\
$4^{*}+6^{*}$ & $2^{*}+4^{*}+6^{*}$ & $2 \cdot 2^{*}+4^{*}+6^{*}$ \\
$3^{*}+7^{*}$ & $2^{*}+3^{*}+7^{*}$ & $2 \cdot 2^{*}+3^{*}+7^{*}$ \\
$1^{*}+9^{*}$ & $1^{*}+2^{*}+9^{*}$ & $1^{*}+2 \cdot 2^{*}+9^{*}$ \\
& $1^{*}+5^{*}+6^{*}$ & $1^{*}+2^{*}+5^{*}+6^{*}$ \\
& $2 \cdot 3^{*}+6^{*}$ & $2^{*}+2 \cdot 3^{*}+6^{*}$ \\
& & $1^{*}+3^{*}+8^{*}$ & $1^{*}+2^{*}+3^{*}+8^{*}$ \\
& & $2^{*}$ & $2^{*}+2^{*}$ \\
& & &
\end{tabular}

\section{REFERENCES}

[B] E. Bertram, On a theorem of Schreier and Ulam for countable permutations, J. Algebra 24 (1973), 316-322.

[Bo] G. Boccara, Cycles comme produit de deux permutations de classes donnees, Discrete Math. 38 (1982), 129-142.

[D1] M. Droste, Products of conjugacy classes of the infinite symmetric groups, Discrete Math. 47 (1983), 35-48.

[D2] _ Cubes of conjugacy classes covering the infinite symmetric groups, Trans. Amer. Math. Soc. 288 (1985), 381-393.

[D3] _ Classes of universal words for the infinite symmetric groups, Algebra Universalis 20 (1985), 205-216.

[DG] M. Droste and R. Göbel, On a theorem of Baer, Schreier and Ulam for permutations, J. Algebra 58 (1979), 282-290.

[Dv] Y. Dvir, Covering properties of permutation groups, Products of Conjugacy Classes in Groups (Z. Arad and M. Herzog, eds.), Lecture Notes in Math., vol. 1112, Springer-Verlag, 1985.

[G] A. B. Gray, Infinite symmetric and monomial groups, Ph.D. Thesis, New Mexico State Univ., Las Cruces, N.M., 1960.

[MO] G. Moran, The algebra of reflections of an infinite set, Notices Amer. Math. Soc. 73T (1973), A 193.

[M1] - The product of two reflection classes of the symmetric group, Discrete Math. 15 (1976), $63-77$.

[M2] , Reflection classes whose cubes cover the alternating group, J. Combin. Theory Ser. A 21 (1976), 1-19.

[M3] - Permutations as products of $k$ conjugate involutions, J. Combin. Theory Ser. A 19 (1975), 240-242.

[M4] - Trees and the bireflection property, Israel J. Math. 41 (1982), 244-260.

[M5] - Of planar Eulerian graphs and permutations, Trans. Amer. Math. Soc. 287 (1985), $323-341$.

Department of Mathematics, University of haifa, Mount Carmel, haifa 31999, ISRAEL 